\title{
INTELLIGENCE ANALYSIS AMONG RURAL LEARNERS IN MOROCCO
}

\author{
Khalid Marnoufi ${ }^{1}$, Bouzekri Touri ${ }^{1}$, Mohammed Bergadi ${ }^{1}$, \& Imane Ghazlane $^{\mathbf{1 , 2}}$ \\ ${ }^{1}$ Multidisciplinary Laboratory of Information, Communication and Education Sciences and Technology \\ (LAPSTICE), Ben M'sik Faculty of Science, Hassan II University of Casablanca, post box 7955, \\ Casablanca (Morocco) \\ ${ }^{2}$ Higher Institute of Nursing and Health Technology Professions Casablanca-Settat, Morocco Street \\ Faidouzi Mohamed, 20250 Casablanca (Morocco)
}

\begin{abstract}
The full scale intelligence quotient is a strong predictor of educational success. The aim of this study was to calculate the full scale intelligence quotient (FSIQ) of Moroccan rural students in the Safi region. the psychometric test Wechsler intelligence scale for children and adolescents - 5th edition WISC V was used by administering the five indexes, namely the verbal comprehension index, visuospatial index, fluid reasoning index, working memory index and processing speed index, to compare them to the average of the Wechsler tests. The study was carried on 104 students (46 girls and 58 boys) between the ages of 12 and 15 years old, $67 \%$ of the students were boarders and $33 \%$ were external students. We administered all 15 subtests of WISC V on each student participating in this study. The results of the participants in this study who are students in public schools in rural areas show a FSIQ by means, and a verbal comprehension index that is higher than the other four indexes in WISC V. In terms of correlations among the five indexes, the results show different types of relationships among the test indexes.
\end{abstract}

Keywords: FSIQ, WISC V, intelligence, learners, rural.

\section{Introduction}

Intelligence tests were first developing in France for the explicit purpose of predicting school success (Binet and Simon, 1904). Then Wechsler's three intelligence tests appeared in the United States and became the most widely used cognitive tests in the world (Camara, Nathan and Puente, 2000; Georgas, van de Vijver, Weiss and Saklofske, 2003; Lichtenberger \& Kaufman, 2009). All three tests are administered individually for adults, adolescents and children. All Wechsler tests have been translated, adapted and standardized in many countries. Since the launch of the Wechsler Bellevue intelligence scale (WB), this rich history of research and clinical applications has undoubtedly contributed to the development of these three tests. From the creation of the Wechsler tests to the latest edition, they based on the g-factor of Spearman, C. (1904). He observed that children's performance on different school subjects was positively correlated.

Thus, the developers of these tests stated that they not only used the general intelligence factor but were also open to cognitive theories to guide creation. Such as use of Cattell-Horn-Carroll theory (CHC). This theory used to classify performance tests to facilitate the interpretation of academic ability and to provide a basis for organizing assessments for those suspected of having a learning disability. In 1993 by Carroll, this theory came together after several legendary analyses in psychology over the past 60 or 70 years concerning the nature, identification and structure of human cognitive abilities and is dividing into three hierarchical levels (Carroll, 1993). The third level is dominated by the general factor (g), which is a common factor among tests after factor analysis by Spearman (1927). In 1941 Raymond Cattell identified two factors of intelligence, fluid intelligence and crystallized intelligence (Gf-Gc), but in 1965 John L. Horn developed this theory from his supervisor, Cattell, through an empirical study in his $\mathrm{PhD}$. Six factors in the first edition and fourteen factors by McGrew. (Quantitative knowledge (Gq), short term memory (Gsm), visual processing (Gv), long term memory (Glr), processing speed (Gs), decision/reaction time) / Speed (Gt), Reading and writing ability (Grw). Auditory Processing (Ga), Domain-specific knowledge (Gkn), Psychomotor Capacity (Gp) and Psychomotor Velocity (Gps) including tactile (Gh), kinesthetic (Gk), and olfactory (Go)) plus the two Cattell-Horn factors (Gf-Gc) that take the second level in CHC theory which are then subdividing into four, then come ten prime factors such as induction (I), language development (LD), lexical knowledge (LK). In the latest update of the CHC model by Schneider and McGrew (2012). 
This study is carried by the latest version of Wechsler Intelligence Scale for Children and Adolescents - 5th edition WISC V. The WISC V is a test of children cognitive abilities and for learning outcomes. It describes the cognitive processes, the theoretical link between cognitive processes and specific academic skills and how to use performance on the WISC-V to generate hypotheses about processing deficits. That considered the score that is most representative of global intellectual functioning (g). Traditionally, FSIQ has been the first score to be considered in profile interpretation. The aim is to calculate the full scale intelligence quotient by the seven subtests of WISC V for Moroccan students in rural areas to measure FSIQ.

\section{Method}

\subsection{Population and procedure}

We sought to administer the test to a sample of families understanding the psychometric analyses of the people in this rural area with the help of the executives and teachers of the regional academy of education and training of the Marrakech-Safi region. The study carried on 104 pupils in rural areas: the participants are (46 girls and 58 boys) between the ages of 12 and 15 years old (mean $=13.11$ months $\& 23$ days with a standard deviation of 01 year, 1 month \& 27 days), 67\% of whom were boarders and $33 \%$ were students who commuted to and from school once a day. We administered all subtests of the Wechsler Intelligence Scale for Children and Adolescents - 5th Edition WISC V on each of the students participating in this study who are enrolling in a rural middle school commune called Khmis Naga in Safi province. We have chosen the samples according to their availability after or before classes, without forgetting students suggested by the general supervisor of the boarding school and who are all boarding students respecting the age order of WISC V. We also carried a parental questionnaire on the educational level of student's parents, uncles and aunts. Without forgetting household income, access to drinking water and electricity, transport and pre-school education.

\subsection{Instrument}

The Wechsler Intelligence Scale for Children and Adolescents - Fifth Edition (WISC - V) administered in the order of the manual. This test of cognitive ability is administered to participants aged 6 years to 16 years and 11 months. It consists of 15 subtests. Ten main sub-tests: similarities, vocabulary, block design, visual puzzles, matrix reasoning, figure weights, digit span, picture span, coding and symbol search plus five additional subtests: information, comprehension, arithmetic, letter-number sequencing, and cancellation. All subtests designed to measure five scales: verbal comprehension, visual spatial, fluid reasoning, working memory and processing speed. The score hierarchically ordered full scale intelligence quotient(FSIQ) is composed of only seven primary subtests and not ten subtests like the fourth version of WISC to measure five scales: verbal comprehension (similarities, vocabulary), visual spatial (block design), fluid reasoning (matrix reasoning, figure weights), working memory (digit span) and processing speed (coding) Wechsler, D. (2016a,b).

\section{Result}

The correlation between the averages of the overall grades of the two semesters of the students' class and the full scale intelligence quotient (FSIQ) for the students is 0.62 . The results of the WISC V psychometric test scores for Moroccan students gave a Cronbach's alpha coefficient of 0.85 in rural areas. Not to mention that girls scored 103.26 in the FSIQ lower than the 104.26 score obtained by boys.

Table 1 presents the mean values and standard deviations of the five indexes and the full scale intelligence quotient (FSIQ) for all participants in this test. We find that the visuospatial index VSI, the fluid reasoning index FRI and the processing speed index PSI are below the mean, while the working memory index WMI is above the mean. Only the verbal comprehension index VCI is slightly above average.

Figure 1. Indexes Scores of rural pupils.

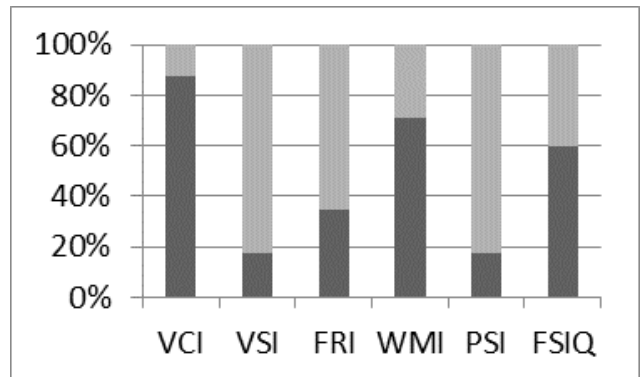


The figure show in black, the percentage of students with index scores greater than or equal to 100 and in grey, the percentage of students with index scores less than 100 .

Note that the difference between the VCI and the closest index WMI is 17 points or one standard deviation. The standard deviation of the two indexes VCI and WMI is different by 7 points. The difference between mean of the WMI and the three indexes VSI, FRI and PSI is between 11 and 15 points or more than one standard deviation. To refine the point about the superiority of the mean VCI over the other mean factor indexes, we note that the figure illustrates the different percentages of children scoring high and low on all indexes and on the full scale intelligence quotient (FSIQ).

Table 1. $M=$ Mean, $S D=$ Standard Deviation, $V C I=$ Verbal Comprehension Index, FRI= Fluid Reasoning Index, $C S=$ class scores.

\begin{tabular}{|c|c|c|c|c|c|c|}
\hline & VCI & VSI & FRI & WMI & PSI & FSIQ \\
\hline M & 122,67 & 92,21 & 94,52 & 106 & 91,17 & 104,05 \\
\hline SD & 19,81 & $\mathbf{9 , 0 7}$ & $\mathbf{1 2 , 0 5}$ & $\mathbf{1 2 , 9 9}$ & $\mathbf{1 1 , 7 5}$ & $\mathbf{1 2 , 3 5}$ \\
\hline
\end{tabular}

A large percentage of the children scored above the theoretical one hundred average in the VCI, WMI and FSIQ which is shown in the figure, whereas less than forty percent of the students scored below the theoretical one hundred average in the VSI, FRI and PSI.

Based on the results of the exploratory factor analysis, a one-factor model was tested with the sample. The two- and three-factor models did not result in saturation coefficients and communities higher than the one-factor model. The confirmatory factor analysis one-factor gave satisfactory adjustment statistics; RMSEA =, 043, CFI =, 967 and TLI =, 956.

The participants in this study compared themselves to the theoretical average 100 in the Full Scale Intelligence Quotient (FSIQ) with a rate of $13.46 \%$ average low, $56.73 \%$ average and $15.38 \%$ average high. For those participants who achieved a high Full Scale Intelligence Quotient (FSIQ), the study recorded $12.5 \%$. As for the high Full Scale Intelligence Quotient (FSIQ), the study recorded only $1.92 \%$ very high according to the Wechsler psychometric test (the Full Scale Intelligence Quotient (FSIQ) equal to or greater than 130 ).

Table 2 shows the matrix of correlations between the five WISC V indexes with the strong and weak positive correlation.

Table 2. Matrix of correlations between the five indexes for the group of children.

\begin{tabular}{|c|c|c|c|c|}
\hline & VCI & VSI & FRI & WMI \\
\hline VSI & $\mathbf{0 , 4 4}$ & & & \\
\hline FRI & $\mathbf{0 , 5}$ & $\mathbf{0 , 5 1}$ & & \\
\hline WMI & $\mathbf{0 , 3 8}$ & $\mathbf{0 , 4}$ & $\mathbf{0 , 5 4}$ & \\
\hline PSI & $\mathbf{0 , 0 2}$ & $\mathbf{0 , 2 6}$ & $\mathbf{0 , 2 8}$ & $\mathbf{0 , 4}$ \\
\hline
\end{tabular}

It can be seen that the correlation between the four indexes VCI, FRI, WMI and VSI are between strong positive relationships and moderate positive relationships but the correlations between PSI with the three indexes are between moderate positive relationship and no or negligible positive relationship.

\section{Discussion}

The full scale intelligence quotient is used to solve school problems among learners. The results of our study are very satisfying because the average value of the full scale intelligence quotient is situated at the theoretical average, which is in contrast with studies already carried on the full scale intelligence quotient in Morocco, which revealed very inferior values. The studied samples showed a correlation between the general averages of the school year and the full scale intelligence quotient values. For students from rural areas there was a strong positive correlation with the reliability of administration given by alpha Cronbach. Girls scored lower in the full scale intelligence quotient which is contradictory to the difference in intelligence of gender despite the number of girls participating in this study being less than half of the entire sample. More generally, IQ is positively correlated with a wide range of life outcomes, including income (Zagorsky, 2007), mental and physical health (Der, Batty, \& Deary, 2009; Gale, Hatch, Batty, \& Deary, 2009), or life expectancy (Batty, Deary, \& Gottfredson, 2007). 
The mean of the verbal comprehension index VCI is high and the standard deviation of this index is higher than the other indexes. It is composed of two subtests similarities and vocabulary with a strong positive correlation between the two subtests. This index is used to measure crystallized intelligence related to the educational level of the child's parents and to the child's environment. However, only $5 \%$ of the sample has parents who were able to receive primary education even from their family circle, not forgetting that not all children were taught in the pre-school period and that the parents' socio-professional category environment is very low based on agriculture. This test, although very successful for children with high potential.

The Visuospatial index VSI requires the child to use his or her visuospatial intelligence. It is composed of two subtests cubes and visual puzzles with a low positive correlation between the two subtests, this index designates the quality of perception and visual organization, motor/visual coordination, the ability to analyze, synthesize, encode and manipulate physically or mentally objects and shapes to give them meaning. The absence of all mental activities and technological means such as computers in this environment with the difficulty of access to electricity does not help develop this intelligence.

The score of the fluid reasoning index (FRI) for the sample is below average so their relationship with three indexes is strong positive relationship only the relationship of this index with the processing speed index is a weak positive relationship. This index is calculated by two subtests Matrices and Balances, it measures the quality of reasoning, the ability to classify and locate in space, and also the ability to solve problems independently of one's knowledge several studies carried locally in Morocco based on tests of fluid intelligence, the studies gave very low results of IQ in Morocco below the average.

The average of the working memory index WMI is above average. This index is calculated by the two subtests Memory of Numbers and Memory of Pictures, which have a low positive correlation. The objective is to use as much information as possible, going as fast as possible. Low scores are obtained in this index which will indicate learning difficulties and more repetitions to record them. The low scores of the children in this index compared with their school performance are evidence of their low academic achievement. It is observed that the links of the processing speed index PSI with the four indexes and the lowest correlations also the score obtained by the students is the lowest compared to the other indexes. It is calculated by the two subtests Code and Symbols with high positive correlation, this index for the speed and accuracy of assimilation of information by a child. Children with low scores in this index showed a delay in processing information. We note that the full scale intelligence quotient is at the average by the WISC V which is different from the studies already carried on Moroccans by Raven's matrices with very low IQ (fluid intelligence) despite the fact that Raven's matrices calculate fluid intelligence.

\section{Conclusion}

The results of this study are dominated by the average for the full scale Intelligence Quotient also for the five indexes of the test, in spite of the problems that surround the life of students in rural areas due to the absence of several essential conditions caused by poverty without forgetting illiteracy in the families. We have noticed that the average of the verbal comprehension index that represents the crystallized intelligence in the study samples that is high in comparison with the other indexes followed by the index of working memory, these two indexes are related to school success. The averages of the three indexes are inferior to the theoretical average for the visuospatial index VSI, the fluid reasoning index FRI and the processing speed index PSI. The problems of education are related to several factors like disorders of communication economic and social state need to be developed by specialists. Rather, the fluid intelligence by fluid reasoning index of WISC V in rural areas for Moroccans is better according to the more than two study of IQ in Morocco by Raven test.

\section{References}

Batty, G. D., Deary, I. J., \& Gottfredson, L. S. (2007). Premorbid (early life) IQ and Later mortality risk: systematic review. Annals of Epidemiology, 17(4), 278-288. https://doi.org/10.1016/j.annepidem.2006.07.010.

Binet, A., \& Simon, T. (1904). Methodes nouvelles pour le diagnostic du niveau intellectual des anormaux. L'Annee Psychologique, 11(1), 191-244. https://doi.org/10.3406/psy.1904.3675.

Camara, W. J., Nathan, J. S., \& Puente, A. E. (2000). Psychological test usage: Implications in professional psychology. Professional Psychology: Research and Practice, 31, 141-154. 
Der, G., Batty, G. D., \& Deary, I. J. (2009). The association between IQ in adolescence and a range of health outcomes at 40 in the 1979 US National Longitudinal Study of Youth. Intelligence, 37(6), 573-580. https://doi.org/10.1016/j.intell.2008.12.002.

Gale, C. R., Hatch, S. L., Batty, G. D., \& Deary, I. J. (2009). Intelligence in childhood and risk of psychological distress in adulthood: The 1958 National Child Development Survey and the 1970 British Cohort Study. Intelligence, 37(6), 592-599. https://doi.org/10.1016/j.intell.2008.09.002.

Georgas, J., van de Vijver, F. J. R., Weiss, L. G., \& Saklofske, D. H. (2003). A cross-cultural analysis of the WISC-III. In J. Georgas, L. G. Weiss, F. J. R. van de Vijver, \& D. H. Saklofske (Eds.), Culture and children's intelligence: Cross-cultural analysis of the WISC-III (pp. 278-313). San Diego, CA: Academic Press.

Lichtenberger, E. O., \& Kaufman, A. S. (2009). Essentials of WAIS-IV Assessment. New York, NY: John Wiley.

Schneider, W. J., \& McGrew, K. S. (2012). The Cattell-Horn-Carroll model of intelligence. In D. P. Flanagan, \& P. L. Harrison (Eds.). Contemporary intellectual assessment (pp. 99-144). (3rd ed.). New York, NY: Guilford Press.

Spearman, C. (1904). "General Intelligence", objectively determined and measured. The American Journal of Psychology, 15, 201-292.

Wechsler, D. (2016a). WISC-V. Echelle d'intelligence de Wechsler pour enfants-5e édition. Paris, France: Pearson France-ECPA.

Wechsler, D. (2016b). WISC-V. Echelle d'intelligence de Wechsler pour enfants-5e édition, manual d'interprétation. Paris, France: Pearson France-ECPA.

Zagorsky, J. L. (2007). Do you have to be smart to be rich? The impact of IQ on wealth, income and financial distress. Intelligence, 35(5), 489-501. https://doi.org/10.1016/j.intell.2007.02.003. 